\title{
Genetic Analysis of Resistance to Leaf Rust and Yellow Rust in Spring Wheat Cultivar Kenya Kongoni
}

V. Calvo-Salazar, International Maize and Wheat Improvement Center (CIMMYT), Apdo. Postal 6-641, 06600 Mexico D.F., Mexico and Colegio de Post-graduados-Genética, Campus Montecillo, Carretera Mexico-Texcoco Km 36.5, Montecillo, Texcoco 56230, Estado de Mexico; R. P. Singh, CIMMYT; J. Huerta-Espino, Campo Experimental Valle de Mexico INIFAP, Apdo. Postal 10, 56230, Chapingo, Edo. de Mexico, Mexico; S. Cruz-Izquierdo, R. Lobato-Ortiz, and S. Sandoval-Islas, Colegio de Post-graduados-Genética, Campus Montecillo; M. Vargas-Hernández, CIMMYT; S. German and P. Silva, National Institute of Agricultural Research (INIA), Route 50 km 11.500, CP 70000, Colonia, Uruguay; and B. R. Basnet, C. X. Lan, and S. A. Herrera-Foessel, CIMMYT

\begin{abstract}
Calvo-Salazar, V., Singh, R. P., Huerta-Espino, J., Cruz-Izquierdo, S., Lobato-Ortiz, R., Sandoval-Islas, S., Vargas-Hernández, M., German, S., Silva, P., Basnet, B. R., Lan, C. X., and Herrera-Foessel, S. A. 2015. Genetic analysis of resistance to leaf rust and yellow rust in spring wheat cultivar Kenya Kongoni. Plant Dis. 99:1153-1160.

The Kenyan wheat (Triticum aestivum L.) 'Kenya Kongoni' exhibits high levels of adult plant resistance (APR) to leaf rust (LR) and yellow rust (YR). We determined the genomic regions associated with LR and YR resistance in a population of 148 recombinant inbred lines generated from a cross between 'Avocet- $Y r A$ ' and Kenya Kongoni. Field experiments to characterize APR to LR and YR were conducted in four and two Mexican or Uruguayan environments, respectively. A linkage map was constructed with 438 diversity arrays technology and 16 simplesequence repeat markers by JoinMap 4.1 software. Genetic analyses showed that resistance to both rusts was determined by four to five

APR genes, including $L r 46 / Y r 29$ and $S r 2 / L r 27 / Y r 30$. Quantitative trait loci (QTL) analysis indicated that pleiotropic APR loci QYLr.cim-1BL corresponding to $\mathrm{Lr} 46 / \mathrm{Yr} 29$ and QYLr.cim-7BL that is a putative novel QTL accounted for 5 to $57 \%$ and 12 to $35 \%$ of the phenotypic variation for resistance to LR and YR, respectively. These loci, in combination with another three LR QTL and two YR QTL, respectively, conferred high levels of resistance to both LR and YR in wheat under Mexican and Uruguayan environments. Among other detected QTL, QLr.cim-1DS, QLr.cim-2BL, and QYLr.icm-7BL may be new loci for APR to both rusts in common wheat.
\end{abstract}

Leaf rust (LR) and yellow rust (YR), caused by Puccinia triticina Erikss. and $P$. striiformis f. tritici Erikss., respectively, are important diseases of wheat worldwide (34). They constantly threaten wheat production in Mexico because of the pathogens' ability to overcome race-specific resistance in new varieties and also because of their ability to quickly reproduce and move over long distances (17). Similarly, in Uruguay, LR is the most widespread and important disease of wheat whereas YR is economically less important $(11,12)$. In Mexico, wheat is grown annually on about 586,000 ha (9), mainly under irrigation in the northwest state of Sonora and in the El Bajio region, both lowland areas (http://www.siap.sagarpa.gob.mx). Losses of up to $63 \%$ from LR and $60 \%$ from YR have been documented in bread wheat crops in those areas $(17,33,38)$. In Uruguay, the total wheat area is 500,000 ha and production is concentrated in the Soriano, Colonia, and Black River Departments (9). Grain yield losses of more than $50 \%$ have been reported from LR in the southwest of this country (11).

The deployment of resistant cultivars is the most efficient way to manage these diseases. The two types of resistance pertaining to LR and YR in wheat are seedling resistance and adult plant resistance (APR). Seedling resistance is usually race specific, governed by single genes often with major effects (18), and is easily overcome by new virulent races. In contrast, APR can be race nonspecific, often controlled by several genes with minor but additive effects $(18,23)$, and usually is believed to be more durable than seedling resistance $(23,41)$.

Thus far, more than 74 LR and 67 YR resistance genes have been cataloged (30). Among these, most are seedling resistance genes and several are not effective against prevalent races in Mexico and

Corresponding authors: C. X. Lan; E-mail: c.lan@cgiar.org; and S. A. Herrera-Foessel, E-mail: sybilherreraf@gmail.com

Accepted for publication 9 February 2015.

http://dx.doi.org/10.1094/PDIS-07-14-0718-RE

(C) 2015 The American Phytopathological Society
Uruguay. Lr34/Yr18/Sr57/Pm38/Ltn1 (22,39), Yr36 (50), Lr46/ Yr29/Sr58/Pm39/Ltn2 (40,57), Sr2/Lr27/Yr30/Pbc1 (28,43), Lr67/ Yr46/Sr55/Pm46/Ltn3 (15), Lr68 (14), Lr74 (U. Bansal, the University of Sydney, personal communication), and $\operatorname{Yr} 54$ (3) confer APR to LR or YR in wheat. Of the preceding genes, $\operatorname{Lr} 34 / \mathrm{Yr} 18 / \mathrm{Sr} 57 /$ Pm38/Ltn1, Lr46/Yr29/Sr58/Pm39/Ltn2, Sr2/Lr27/Yr30/Pbc1, and Lr67/Yr46/Sr55/Pm46/Ltn3 are of particular interest because they are pleiotropic to multiple diseases, including stem rust and powdery mildew (caused by $P$. graminis f. sp. tritici and Blumeria graminis f. sp. tritici, respectively) and are also associated with morphological traits—leaf tip necrosis (LTN) or pseudo black chaff_-often used as visual markers to detect the presence of these resistances. In addition, around 80 LR (26) and 140 YR (35) quantitative trait loci (QTL) have been mapped in wheat. Many of these QTL are located in the same genetic regions for both diseases and are likely to be potential pleiotropic APR loci in wheat (26), such as the QTL on chromosomes 1BS, 2AL, 2BS, 2DL, 5AL, 5BL, 6AL, and 7BL that confer resistance to LR, YR, and powdery mildew simultaneously (26).

'Kenya Kongoni' is an International Maize and Wheat Improvement Center (CIMMYT) germplasm-derived spring wheat cultivar released in Kenya by the Kenya Agricultural Research Institute in 1981. It confers high levels of resistance to LR and YR in Mexico and to LR in Uruguay and manifests strong LTN at the adult plant stage. It does not carry the APR gene Lr34/Yr18/Sr57/Pm38/Ltn1, based on the molecular marker test with $c s L V 34$ (21) and the gene-specific marker (24), nor does it carry Lr67/Yr46/Sr55/Pm46/Ltn3, based on the gene-specific marker (E. S. Lagudah, personal communication).

The objective of our study was to investigate the genetic basis of resistance to LR and YR in Kenya Kongoni by using a recombinant inbred line (RIL) population from its cross with susceptible parent 'Avocet- $Y r A$ ' and to identify the genomic regions associated with resistance to the two rust diseases.

\section{Materials and Methods}

Plant materials. A population of $148 \mathrm{~F}_{5}$ RILs was developed from the cross of the susceptible parent Avocet- $Y r A$ with resistant parent 
Kenya Kongoni. Avocet-YrA is a reselection line from the Australian 'Avocet' that lacks the temporarily designated resistance gene YrA. Kenya Kongoni (pedigree: CI8154/2*FR//3*ROM/4/WIS245/ II50.17//CI8154/2*FR/3/TOB66) is susceptible to current Mexican races of LR and YR at seedling stage but resistant at adult plant stage, whereas Avocet-YrA is susceptible to these races at all growth stages. The population was generated through single-seed descent, where one random spike was harvested in each generation and advanced to the next generation by sowing as hill plots. The same-single seed source of the $\mathrm{F}_{4}$-derived $\mathrm{F}_{5} \mathrm{RIL}$ and parents were used in all experiments.

Field evaluation. The 148 RILs and parents were evaluated for LR resistance at El Batán, Mexico, during 2011 (hereafter referred to as the LR2011 experiment), at Ciudad Obregón, Mexico, during 2011-12 and 2012-13 (hereafter referred to as the LR2012 and LR2013 experiments), and at La Estanzuela, Uruguay, during 2012 (hereafter referred to as the LR2012U experiment). Phenotyping for YR was conducted at Toluca, Mexico, during 2011 and 2012 (hereafter referred to as the YR2011 and YR2012 experiments). The mapping population was evaluated using an augmented design in the LR2012, LR2013, and YR2012 experiments, in which 28 RILs and two parents were replicated twice and randomly distributed among the whole population. The LR2011, LR2012U, and YR2011 field experiments were conducted without replication.

Field plots consisted of $0.7-\mathrm{m}$ paired rows with approximately 60 plants of each line. 'Morocco' and a mixture of six susceptible wheat lines derived from an 'Avocet/Attila' cross were used as LR and YR spreaders, respectively, in LR2011, LR2012, YR2011, and YR2012 field trials, whereas the YR differential line 'Avocet+Yr24/Yr26', susceptible to LR but resistant to YR, was used as an LR spreader in the LR2013 experiment. A mixture of Morocco, 'Thatcher', and 'Little Club' was used as the LR spreader in Uruguay under natural epidemics. Spreaders were planted around the experimental area and as hill plots in the middle of a $0.3-\mathrm{m}$ pathway on one side of each experimental plot. In Mexico, the LR epidemic was initiated by spraying the spreaders with an equal urediniospore mixture of $P$. triticina races $\mathrm{MBJ} / \mathrm{SP}$ and $\mathrm{MCJ} / \mathrm{SP}$ suspended in Sotrol oil (chempoint.com). The avirulence/virulence formula of MBJ/SP and MCJ/SP were reported in a previous study (14). The only difference between the two races is that MCJ/SP is completely virulent against $L r 26$, whereas $\mathrm{MBJ} / \mathrm{SP}$ is partially virulent against this gene. Similarly, a mixture of $P$. striiformis isolates Mex96.11 and Mex08.13 was used to initiate the YR epidemics. Mex96.11 is virulent to $\operatorname{Yr} 27$ and avirulent to $\operatorname{Yr} 31$, whereas Mex08.13 is the opposite. In the present study, the resistant parent
Kenya Kongoni was susceptible to all of these LR and YR races in the seedling stage but resistant in the adult plant stage. Disease severities (DS) on parents and RILs were recorded three times in 2011, 2012, and 2013 using the Modified Cobb's scale (32). The first note was recorded when the susceptible parent Avocet-YrA displayed approximately $80 \%$ severity, and thereafter continued at weekly intervals until severity reached 90 to $100 \%$. For multiple disease readings, area under disease progress curve (AUDPC) values were calculated using the method suggested by Bjarko and Line (4).

Genetic and statistical analysis. The number of APR genes against LR and YR in the Avocet/Kenya Kongoni population was estimated using Mendelian segregation analysis $(20,44)$, where RILs were classified into three phenotypic categories: homozygous parental type resistant (HPTR), homozygous parental type susceptible (HPTS), and lines with responses different from those of the two parents (OTHER) based on disease severities and infection responses. The observed frequencies for each category (HPTR, HPTS, and OTHER) were tested against the expected frequencies for different numbers of additive genes using $\chi^{2}$ analysis. Moreover, the minimum number of APR genes was also estimated for the three experiments grown under an augmented design by using the quantitative approach described by Wright (59) and Mulitze and Baker (31) as $n=(\mathrm{GR})^{2} / 4.57\left(\sigma^{2} \mathrm{~g}\right)$, where genotypic range $(\mathrm{GR})=$ phenotype range $\times$ broad-sense heritability $\left(h^{2}\right)$, and $\sigma^{2} \mathrm{~g}=$ genetic variance of the RILs. Analysis of variance (ANOVA) was used to calculate $h^{2}$ and $\sigma^{2} \mathrm{~g}$ in the augmented design experiment. In order to get a single value for each entry to be used in QTL mapping and phenotypic correlation analyses, the partially replicated designs were analyzed through the mixed-models framework using the Mixed Procedure in SAS (SAS, Institute, Cary, NC) to calculate the best linear unbiased estimators or adjusted means whereas, for the RILs without replication, the final disease severities (FDS) in each environment were used directly.

Molecular analysis and genetic linkage map construction. The CTAB method was used for the DNA extractions of parents and RILs, with approximately 20 plants per line. The two parents and RILs were analyzed using 1,450 diversity arrays technology (DArT) markers and 16 simple-sequence repeat (SSR) primers for the polymorphism survey. In addition, two cleaved amplified polymorphic sequence (CAPS) markers, $c s L V 46 G 22$ and $c s S r 2$, associated with $\operatorname{Lr} 46$ (E. S. Lagudah, personal communications) and $\mathrm{Sr} 2$ (47), respectively, were used for genotyping the RIL population. In total, 455 polymorphic markers were used to construct the linkage maps with Joinmap 4.1 (51). Linkage maps were graphically visualized

Table 1. Final disease severities of parents and an Avocet- $\mathrm{YrA} /$ Kenya Kongoni recombinant inbred line (RIL) population evaluated for leaf rust and yellow rust resistance five and two environments, respectively, and estimated number of resistance genes based on the Wright (59) quantitative method

\begin{tabular}{|c|c|c|c|c|c|c|}
\hline \multirow[b]{2}{*}{ Parent, parameter } & \multicolumn{3}{|c|}{ Leaf rust severity, \% (Mexico) } & \multirow{2}{*}{$\frac{\text { Leaf rust severity, \% (Uruguay) }}{2012^{\mathrm{d}}}$} & \multicolumn{2}{|c|}{ Yellow rust severity, \% (Mexico) } \\
\hline & $2011^{a}$ & $2012^{b}$ & $2013^{c}$ & & $2011^{e}$ & $2012^{\mathrm{f}}$ \\
\hline Avocet & 90 & 90 & 70 & 90 & 90 & 80 \\
\hline Kenya Kongoni & 10 & 5 & 5 & 5 & 5 & 5 \\
\hline Population mean & 52 & 41 & 42 & 51 & 47 & 37 \\
\hline Population range: low & 10 & 5 & 1 & 0 & 5 & 5 \\
\hline Population range: high & 100 & 100 & 90 & 99 & 100 & 90 \\
\hline Entry variance $\left(\sigma_{\mathrm{g}}^{2}\right)$ & $\ldots$ & 578.5 & 272.4 & $\ldots$ & $\ldots$ & 331.5 \\
\hline Residual variance $\left(\sigma_{\mathrm{e}}^{2}\right)$ & $\ldots$ & 34.8 & 26 & $\ldots$ & $\ldots$ & 31.9 \\
\hline Heritability $\left(h^{2}\right)$ & $\ldots$ & 0.94 & 0.91 & $\ldots$ & $\ldots$ & 0.91 \\
\hline Estimated number of genes ${ }^{\mathrm{g}}$ & $\ldots$ & 3 & 5 & $\ldots$ & $\ldots$ & 3.9 \\
\hline
\end{tabular}

${ }^{a}$ Final percent leaf rust severity at El Batán, 2011 season.

b Final percent leaf rust severity at Ciudad Obregón, 2011-12 season.

c Final percent leaf rust severity at Ciudad Obregón, 2012-2013 season.

${ }^{\mathrm{d}}$ Final percent leaf rust severity at La Estanzuela, 2012 season.

e Final percent yellow rust severity at Toluca, 2011 season.

${ }^{\mathrm{f}}$ Final percent yellow rust severity at Toluca, 2012 season.

g Number of segregating genes estimated through the quantitative method. 
with MapChart (53). FDS and AUDPC from each experiment for LR and YR were used for QTL mapping by composite interval mapping (CIM) with Windows QTL Cartographer v2.5 software (54). To declare a significant QTL, 1,000 permutation tests were carried out and a logarithm of odds (LOD) threshold was set at $P=0.05$ for each trait. The percentage of phenotypic variance explained $\left(R^{2}\right)$ by individual QTL and additive effects at the LOD peaks were detected by CIM with backward and forward regression method with QTL Cartographer. A wheat consensus genetic map and physical bin location of DArT markers $(10,16,46,56)$ were used to assign marker orders and the chromosomal position of linkage groups. QTL names were designated following the recommended practice (http://wheat.pw.usda.gov/ ggpages/wgc/98/Intro.htm).

\section{Results}

Phenotypic evaluation. The FDS of Kenya Kongoni was 5\% for LR at the adult plant stage whereas, for Avocet-YrA it ranged between 70 and $90 \%$, depending on the site (Table 1). The mean LR FDS ranged between 41 and $51 \%$ for RILs in the three environments (Table 1). The frequency distributions of RILs for LR severities were continuous in both Mexican and Uruguayan environments (Fig. 1A), indicating polygenic inheritance of APR. Approximately three to five LR APR genes were estimated based on both Wright's method (modified by Mulitze and Baker [31]) (Table 1) and the Mendelian segregation analysis (Table 2).

The FDS of Kenya Kongoni was also 5\% for YR in the two field experiments whereas, for Avocet- $Y r A$ it ranged between 80 and $90 \%$ (Table 1). The mean YR FDS ranged from 37 to $47 \%$ for the RILs (Table 1). The frequency distribution of RILs for YR severity was continuous in the two field experiments (Fig. 1B). Genetic analysis showed that four to five APR genes confer YR resistance in the Avocet-YrA/Kenya Kongoni RIL population, based on the two genetic methods used (Tables 1 and 2).

The Pearson's correlation coefficient for LR FDS between each environment ranged from 0.62 to $0.80(P<0.0001)$ and the correlation for YR was $0.82(P<0.0001)$. Significant correlations were also detected between LR and YR FDS among different environments $(r=0.35$ to $0.64, P<0.0001)$ (Table 3 ). The $h^{2}$ of FDS was 0.94, 0.91, and 0.91 in LR2012, LR2013, and YR2012, respectively, whereas the variation among RILs was 578.5, 272.4, and 331.5, respectively, based on ANOVA (Table 1).

QTL mapping for pleiotropic APR to LR and YR. Two pleiotropic APR loci against LR and YR were detected in the Avocet-YrA/ Kenya Kongoni RIL population. The first QTL, QYLr.cim-1BL, was mapped in the same region as $L r 46 / Y r 29$ based on the neardiagnostic marker csLV46G22. It explained 12 to $57 \%$ and 25 to $35 \%$ of variation for LR and YR severities, respectively, and was flanked by markers $w P t-1770$ and $c s L V 46 G 22$ (Table 4; Fig. 2A). Another locus, QYLr.cim-7BL, detected on the long arm of chromosome $7 \mathrm{~B}$ in the interval of molecular markers $w P t-9925$ and $w P t$ 8938, explained 5 to $13 \%$ of LR and $12 \%$ of YR variance (Table 4; Fig. 2B). These two pleiotropic APR QTLs were derived from the resistant parent Kenya Kongoni.

QTL mapping for APR to LR. Four additional QTL for LR resistance were detected in the Avocet- $Y r A /$ Kenya Kongoni population. The first QTL, QLr.cim-1DS, was located on the short arm of chromosome $1 \mathrm{D}$ and was flanked by DArT markers $w P t-3738$ and $r P t-4471$. It explained 6 to $21 \%$ of LR variance (Table 4 ; Fig. 2C). The second QTL, QLr.cim-2BL, detected in LR2011 and LR2013, explained 6 to $21 \%$ of the variance for LR severity. It was located between DArT markers $w P t-6174$ and $w P t-8548$ (Table 4; Fig. 2D). The third QTL, QLr.cim-3BS, was flanked by molecular markers $w P t-5209$ and $c s S r 2$ and explained 5 to $10 \%$ of the LR variance in adult plants (Table 4; Fig. 2E). The fourth QTL, QLr.cim-5AC, was located near the centromere of chromosome $5 \mathrm{~A}$ and flanked by DArT markers $w P t-3187$ and $w P t-7769$. It was significant only in LR2011. Except for QLr.cim-5AC, which came from the susceptible parent Avocet- $\mathrm{YrA}$, the other QTL were derived from the resistant parent Kenya Kongoni.
QTL mapping for APR to YR. Three minor QTL for YR resistance were mapped in the Avocet- $Y r A /$ Kenya Kongoni population. The first QTL, QYr.cim-2AS, was detected only in YR2011. It was flanked by Xbarc124 and $w P t-1722$ and explained 5 to $6 \%$ of the YR variance for YR severity (Table 4; Fig. 2F). The second QTL, QYr.cim-2BS, was detected in both YR environments and mapped on the short arm of chromosome $2 \mathrm{~B}$ at the interval of $w P t-1813$ and $w P t-8072$. It explained 7 to $12 \%$ of the variation (Table 4; Fig. 2G). QYr.cim-5BL was only detected in YR2011 and flanked by molecular markers Xbarc59 and $w P t$ 3922. It explained 6 to $8 \%$ of YR variance (Table 4; Fig. 2H). All three QTL were derived from the resistant parent Kenya Kongoni.

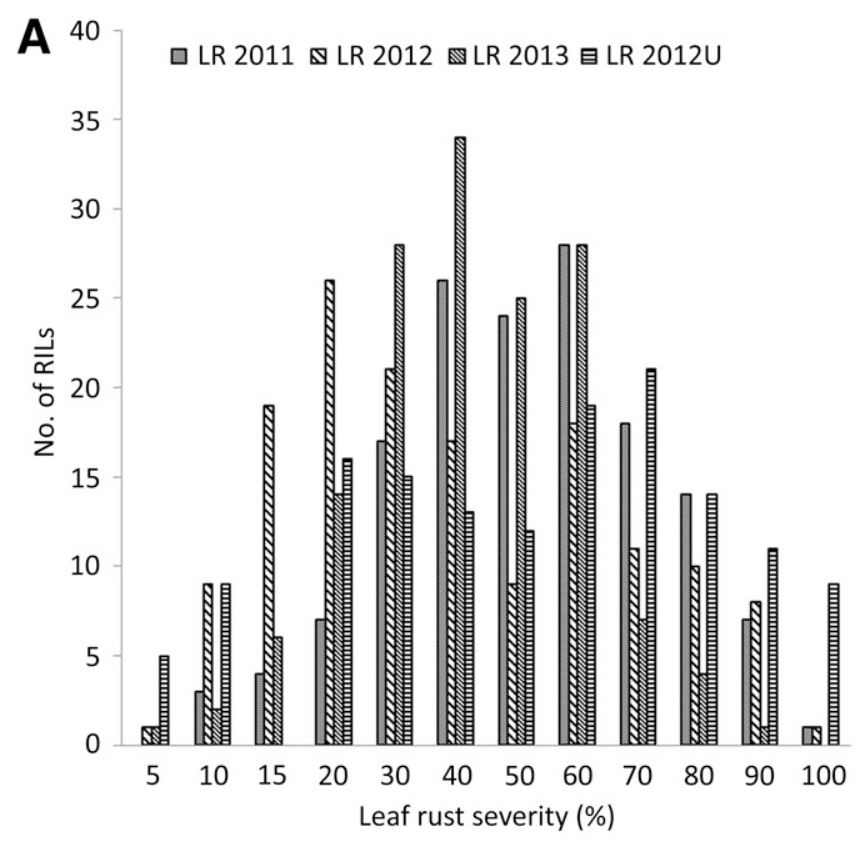

B

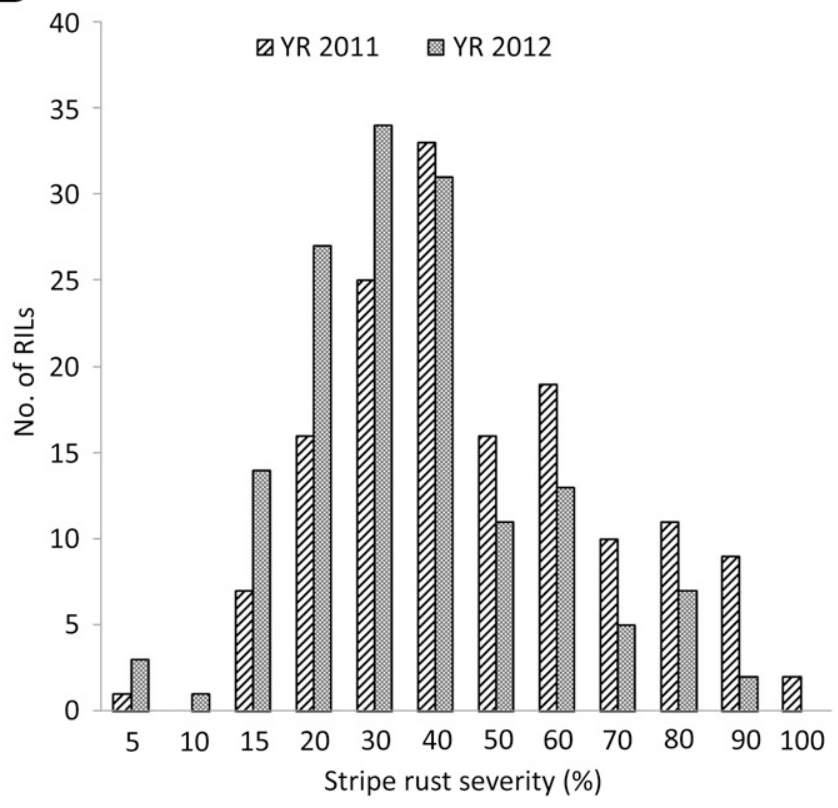

Fig. 1. Frequency distributions for final disease severity in Avocet-YrA/Kenya Kongoni recombinant inbred lines (RILs) for $\mathbf{A}$, leaf rust severity in field trials in Mexico (El Batán, 2011 and Ciudad Obregón, 2012-13) and Uruguay (La Estanzuela, 2012) and for B, yellow rust severity in Mexico (Toluca, 2011 and 2012). 


\section{Discussion}

Using conventional methods to estimate genes, resistance in Kenya Kongoni to LR and YR was found to be governed by four to five APR genes, which agrees with the QTL detected through molecular mapping. QTL on chromosomes 1BL and 3BS are most likely the known pleotropic resistance loci $L r 46 / Y r 29 / S r 58 / P m 39$ and $\mathrm{Sr} 2 /$ $L r 27 / Y r 30$, based on the closely linked molecular markers. Interestingly, the 3BS QTL caused a significant reduction in LR severity but had no significant effect on YR.

The 1BL QTL corresponding to pleiotropic APR gene Lr46/Yr29/ Sr58/Pm39 had the most significant effect in reducing LR and YR severities. This gene provided relatively high levels of protection for LR and YR in all environments and explained the expression of LTN in the population. This locus has also been reported in other populations $(3,25,36,37,42,57,58)$.

Another pleiotropic QTL associated with resistance to LR and YR was found on chromosome 7BL between markers $w P t-9925$ and $w P t-8938$, temporarily designated as QYLr.cim-7BL. Crossa et al. (7) reported that the marker $w P t-9925$ may be associated with the YR APR gene Yr39. Lin and Chen (27) identified Yr39 closely linked with molecular marker $X g w m 131$ in spring wheat 'Alpowa'. This marker was analyzed in the parental lines Avocet-YrA and Kenya Kongoni but was found to be monomorphic. HerreraFoessel et al. (14) mapped the slow-rusting APR gene Lr68 in 'Parula' on chromosome arm 7BL at a distance of 7.5 centimorgans (cM) from the marker Xgwm146, which was tightly linked to the race-specific resistance gene $L r 14 b$. Both Avocet-YrA and Kenya Kongoni possessed the negative allele of the $c s 7 B L N L R R$ marker, known to be tightly linked with $L r 68 / L r 14 b$. In their study with 'Attila' wheat, Rosewarne et al. (37) found a QTL with significant effect on chromosome 7BL between markers Xwmc273-Xgwm146 and XP32/M59-Xgwm344 for LR and YR resistance, respectively. The SSR markers Xgwm344 and Xgwm146, also known to be associated with $\mathrm{Lr} 68 / \mathrm{Lrl}$ H bere used to genotype the parents and RILs and were found to be located at a distance of 27.3 and $32.0 \mathrm{cM}$, respectively, from the $Q Y L$ r.cim-7BL. Due to the long distances of the Lr68/Lr14b linked markers from QYLr.cim-7BL in the Avocet-YrA/ Kenya Kongoni RIL population, it is likely that this QTL is an uncharacterized pleiotropic slow-rusting APR gene that confers resistance to LR and YR. Moreover, $L r 68 / L r 14 b$ is not known to confer APR to YR to date.

The LR QTL QLr.cim-1DS on chromosome 1DS between markers $w P t-3738$ and $w P t-4471$ was more significant in Uruguay, with an LOD of 10 and PVE of 10\%. In Mexico in 2011, the same markers were associated with a QTL for LR resistance but with a lower LOD and PVE of 3 to 5 and $5.6 \%$, respectively. The DArT marker $w P t-3738$ was associated with grain yield (7) and the LR race-specific resistance gene $L r 42$. The gene was flanked by markers Xwmc432 and Xcdf15 and mapped on chromosome 1DS (49). Basnet et al. (3) also found Lr42 in 'Quaiu 3' wheat using the same SSR marker, Xwmc432. This marker was used in the parents of our study and turned out to be monomorphic. AvocetYrA and Kenya Kongoni were susceptible to the races used in the present study at the seedling stage and, therefore, are not expected to carry $\operatorname{Lr} 42$.

The LR QTL QLr.cim-2BL was located in the interval of $w P t-6174$ and $w P t-8548$. Previous studies also have shown LR QTL on 2BL, indicating that this region is important for slowrusting resistance to LR. Chromosome $2 \mathrm{BL}$ is of interest in this study because it showed an LOD and PVE of 3 to 13 and 6 to $20 \%$, respectively. Rosewarne et al. (37) found a significant QTL on chromosome 2BL for YR derived from Avocet-YrA flanked by the SSR markers Xgwm1027 and Xgwm61. The QTL on 2BS in the present study was derived from Kenya Kongoni. It is difficult to clarify the relationship between these two QTL because the common molecular marker was not available; however, this chromosomal region requires further investigation.

The QTL QLr.cim-3BS for LR resistance on 3BS, detected in the Avocet- $Y r A /$ Kenya Kongoni population, was associated with the tightly linked genes Sr2/Lr27/Yr30. The P. triticina races used in our experiments were virulent on the complementary leaf rust resistance genes $L r 27+L r 31$; hence, the QTL effect is unlikely to be associated with $L r 27$. Previous studies identified QTL for LR on chromosome $3 \mathrm{BS}(8,19,36,43,48)$. Spielmeyer et al. (47)

Table 3. Phenotypic correlations for final leaf rust severity in Mexico (El Batán, 2011; Ciudad Obregón, 2011-12 and 2012-13) and Uruguay (La Estanzuela, 2012) and yellow rust severity in Mexico (Toluca, 2011 and 2012) for an Avocet-YrA/Kenya Kongoni RIL population ${ }^{\mathrm{a}}$

\begin{tabular}{lccccc}
\hline Environment & LR2011 $^{\text {b }}$ & LR2012 $^{\mathbf{c}}$ & LR2013 $^{\mathbf{d}}$ & LR2012U $^{\mathbf{e}}$ & YR2011 $^{\mathbf{f}}$ \\
\hline LR2012 & $0.75^{* *}$ & $\ldots$ & $\ldots$ & $\ldots$ & $\ldots$ \\
LR2013 & $0.72^{* *}$ & $0.80^{* *}$ & $\ldots$ & $\ldots$ & $\ldots$ \\
LR2012U & $0.66^{* *}$ & $0.62^{* *}$ & $0.65^{* *}$ & $\ldots$ & $\ldots$ \\
YR2011 & $0.53^{* *}$ & $0.61^{* *}$ & $0.57^{* *}$ & $0.35^{* *}$ & $\ldots$ \\
YR2012 & $0.56^{* *}$ & $0.64^{* *}$ & $0.64^{* *}$ & $0.36^{* *}$ & $0.82^{* *}$ \\
\hline
\end{tabular}

a Asterisks (**) indicate means significant at $P<0.0001$.

${ }^{b}$ Final leaf rust severity at El Batán, 2011 season.

c Final leaf rust severity at Ciudad Obregón, 2011-12 season.

d Final leaf rust severity at Ciudad Obregón, 2012-13 season.

e Final leaf rust severity at La Estanzuela, 2012 season.

${ }^{\mathrm{f}}$ Final yellow rust severity at Toluca, 2011 season.

g Final yellow rust severity at Toluca, 2012 season.

Table 2. Estimated number of resistance genes through Mendelian segregation analysis for adult plant resistance to leaf rust and yellow rust in 148 Avocet- $\mathrm{YrA} /$ Kenya Kongoni $\mathrm{F}_{5}$ recombinant inbred lines (RILs)

\begin{tabular}{|c|c|c|c|c|c|c|}
\hline \multirow[b]{3}{*}{ Response category ${ }^{d}$} & \multicolumn{6}{|c|}{ Number of RILs } \\
\hline & \multicolumn{3}{|c|}{ Leaf rust (Mexico) ${ }^{\mathbf{a}}$} & \multirow{2}{*}{$\frac{\text { Leaf rust (Uruguay) }}{2012}$} & \multicolumn{2}{|c|}{ Yellow rust ${ }^{\mathrm{c}}$ (Mexico) } \\
\hline & 2011 & 2012 & 2013 & & 2011 & 2012 \\
\hline HPTS & 6 & 8 & 6 & 8 & 2 & 2 \\
\hline HPTR & 2 & 1 & 2 & 1 & 2 & 2 \\
\hline OTHER & 139 & 138 & 135 & 138 & 143 & 142 \\
\hline Missing & 1 & 1 & 5 & 1 & 1 & 2 \\
\hline Total & 148 & 148 & 148 & 148 & 148 & 148 \\
\hline Number of genes & 4 & 4 & 4 & 4 & 5 & 5 \\
\hline$P$ value & 0.32 & 0.09 & 0.35 & 0.09 & 0.92 & 0.92 \\
\hline
\end{tabular}

a Based on leaf rust severity recorded in three seasons (El Batán, 2011; Ciudad Obregón, 2011-12 and 2012-13).

$\mathrm{b}$ Based on leaf rust severity recorded at La Estanzuela, 2012 season.

c Based on yellow rust severity recorded at Toluca, 2011 and 2012 seasons.

${ }^{\mathrm{d}}$ HPTS $=$ homozygous parental type susceptible, HPTR = homozygous parental type resistant, and OTHER = lines with responses different from those of the parents. $P$ value is for the $\chi^{2}$ test. Expected ratios of RILs grouped under HPTR, HPTS, and OTHER are 0.037:0.037:0.926 and 0.016:0.016:0.968 for segregation of four and five additive genes, respectively, in the $\mathrm{F}_{4}$-derived $\mathrm{F}_{5}$ generation, assuming that HPTR lines were derived from plants that were homozygous for all resistance genes, HPTS lines were derived from plants where all resistance genes were absent, and lines grouped as OTHER were derived from plants with all other possible combinations of resistance genes. 
determined that a 120-bp allele of the microsatellite locus Xgwm533 was linked to $S r 2$ in several lines. Mago et al. (28) developed a CAPS marker, $c s S r 2$, associated with the presence or absence of the gene, which was also used in this study. The QLr.cim-3BS effects were significant in Mexico and Uruguay and similar in all experiments. In contrast, the effect of minor APR gene $\mathrm{Yr} 30$ $(48,57)$ was not significant in the Avocet-YrA/Kenya Kongoni population.

A minor YR QTL $Q Y r$.cim-2AS was detected in Kenya Kongoni only during YR2011. It was flanked by molecular markers Xbarc124 and $w P t-1722$ with an LOD of 3 and PVE of 5 to 6\%. Hao et al. (13) detected a QTL for YR resistance in U.S. wheat line Pioneer 26R61 at a similar chromosomal position. It was flanked by SSR markers Xbarc 124 and Xgwm359 and explained 23 to $24 \%$ of YR variation in adult plant stage. In addition, several QTL have been reported in this region in wheat lines Y16DH70 (1), Recital (8), Camp Remy
(29), Stephens (52), Kukri (2), and T. monococcum (5). The QTL in T. monococcum should be different from QYr.cim-2AS, based on the origin, but the relationship between $Q Y$ r.cim- $2 A S$ and others needs further investigation.

A QTL associated with moderately effective YR resistance was located on chromosome 2BS. The QYr.cim-2BS is positioned between markers $w P t-1813$ and $w P t-6158$ and had a range of 3 to 5 LOD and 6 to $10 \%$ PVE. This region is rich in race-specific resistance genes, including $\mathrm{Yr} 31$ (45) and $\mathrm{Yr} 27$ (55), that show an intermediate infection type when scored in seedling tests against avirulent races and also confer moderate to high levels of resistance when present alone in adult plants with avirulent races. Based on seedling tests (results not shown), we can conclude that the genes $Y r 27$ and $Y r 31$ were absent in Kenya Kongoni, due to its high infection types in seedlings with races avirulent to these genes.

Table 4. Position and effect of quantitative trait loci (QTL) detected for adult plant resistance (APR) to leaf rust and yellow rust using final disease severity (FDS) and area under the disease progress curve (AUDPC) through composite interval mapping (CIM) by QTL Cartographer in the Avocet-YrA/Kenya Kongoni RIL population

\begin{tabular}{|c|c|c|c|c|c|c|c|}
\hline Environment & $\mathbf{Q T L}^{\mathbf{a}}$ & Position $^{b}$ & Left marker & Right marker & LOD $^{\mathbf{c}}$ & $R^{2}(\%)^{\mathrm{d}}$ & Add $^{e}$ \\
\hline \multirow[t]{4}{*}{ LR11 FDS } & QLr.cim-1BL & 12 & $w P t-1770$ & $\operatorname{csLV} 46 G 22$ & 14 & 24 & -10 \\
\hline & QLr.cim-1DS & 0 & $w P t-3738$ & $r P t-4471$ & 4 & 6 & -5 \\
\hline & QLr.cim-2BL & 0 & $w P t-6174$ & $w P t-8548$ & 12 & 21 & -9 \\
\hline & QLr.cim-5AC & 36 & $w P t-3187$ & $w P t-7769$ & 3 & 5 & 4 \\
\hline \multirow[t]{5}{*}{ LR11AUDPC } & QLr.cim-1BL & 12 & $w P t-1770$ & $\operatorname{csLV} 46 G 22$ & 16 & 28 & -34 \\
\hline & QLr.cim-1DS & 0 & $w P t-3738$ & $r P t-4471$ & 5 & 7 & -17 \\
\hline & QLr.cim-2BL & 0 & $w P t-6174$ & $w P t-8548$ & 12 & 20 & -28 \\
\hline & QLr.cim- $2 D L$ & 12 & $w P t-668017$ & $w P t-666518$ & 3 & 5 & -14 \\
\hline & QLr.cim-5AC & 37 & $w P t-3187$ & $w P t-3884$ & 4 & 5 & 15 \\
\hline \multirow[t]{3}{*}{ LR12 FDS } & QLr.cim-1BL & 22 & csLV46G22 & $w P t-729773$ & 20 & 51 & -20 \\
\hline & QLr.cim-3BS & 5 & $w P t-5209$ & $\operatorname{csSr} 2$ & 3 & 5 & -6 \\
\hline & QLr.cim-7BL & 12 & $w P t-9925$ & $w P t-0194$ & 3 & 6 & -7 \\
\hline \multirow[t]{3}{*}{ LR12 AUDPCg } & QLr.cim-1BL & 23 & csLV46G22 & $w P t-729773$ & 25 & 57 & -111 \\
\hline & QLr.cim-3BS & 5 & $w P t-5209$ & $\operatorname{csSr} 2$ & 6 & 8 & -41 \\
\hline & QLr.cim-7BL & 12 & $w P t-9925$ & $w P t-0194$ & 4 & 7 & -38 \\
\hline \multirow[t]{4}{*}{ LR12Uh } & QLr.cim-1BL & 24 & csLV46G22 & $w P t-729773$ & 5 & 12 & -11 \\
\hline & QLr.cim-1DS & 0 & $w P t-3738$ & $r P t-4471$ & 11 & 21 & -14 \\
\hline & QLr.cim-3BS & 2 & $w P t-5209$ & $c s S r 2$ & 5 & 10 & -10 \\
\hline & QLr.cim-7BL & 32 & $w P t-8938$ & $w P t-9013$ & 7 & 13 & -12 \\
\hline \multirow[t]{2}{*}{ LR13 FDS } & QLr.cim-1BL & 11 & $t P t-6091$ & $w P t-1770$ & 9 & 24 & -10 \\
\hline & QLr.cim-7BL & 0 & $w P t-8981$ & $w P t-2273$ & 3 & 5 & -5 \\
\hline \multirow[t]{3}{*}{ LR13 AUDPC ${ }^{\mathrm{i}}$} & QLr.cim-1BL & 13 & $w P t-1770$ & $w P t-734314$ & 13 & 31 & -63 \\
\hline & QLr.cim-2BL & 1 & $w P t-6174$ & $w P t-8548$ & 3 & 6 & -28 \\
\hline & QLr.cim-2DL & 32 & $w P t-2781$ & Xbarc159 & 3 & 6 & -27 \\
\hline \multirow[t]{4}{*}{ YR11 FDS } & QYr.cim-1BL & 12 & $w P t-1770$ & csLV46G22 & 17 & 35 & -13 \\
\hline & QYr.cim-2AS & 0 & Xbarc124 & $w P t-1722$ & 3 & 5 & -5 \\
\hline & QYr.cim-2BS & 15 & $w P t-1813$ & $w P t-4613$ & 4 & 7 & -6 \\
\hline & QYr.cim-5BL & 135 & barc59 & $w P t-3922$ & 4 & 8 & -6 \\
\hline \multirow[t]{4}{*}{ YR11 AUDPC $^{j}$} & QYr.cim-1BL & 13 & $w P t-1770$ & csLV46G22 & 15 & 32 & -142 \\
\hline & QYr.cim-2AS & 0 & Xbarc124 & $w P t-1722$ & 3 & 6 & -59 \\
\hline & QYr.cim-2BS & 9 & $w P t-1813$ & $w P t-4613$ & 5 & 8 & -71 \\
\hline & QYr.cim-5BL & 135 & Xbarc59 & $w P t-3922$ & 3 & 6 & -64 \\
\hline \multirow[t]{3}{*}{ YR12 FDS } & QYr.cim-1BL & 11 & tPt-6091 & $\operatorname{csLV} 46 G 22$ & 10 & 25 & -11 \\
\hline & QYr.cim-2BS & 21 & $w P t-6158$ & $w P t-5188$ & 4 & 8 & -6 \\
\hline & QYr.cim-7BL & 12 & $w P t-9925$ & $w P t-744632$ & 5 & 12 & -8 \\
\hline \multirow[t]{3}{*}{ YR12 AUDPC } & QYr.cim-1BL & 11 & $t P t-6091$ & $\operatorname{csLV} 46 G 22$ & 14 & 34 & -143 \\
\hline & QYr.cim-2BS & 29 & $w P t-3949$ & $w P t-8072$ & 5 & 12 & -86 \\
\hline & QYr.cim-7BL & 11 & $w P t-9925$ & $w P t-0194$ & 6 & 12 & -85 \\
\hline
\end{tabular}

a QTL that extend across single one-log support confidence intervals were assigned the same symbol.

b Peak position in centimorgans from the first linked marker of the relevant linkage group.

c Logarithm of odds (LOD) score based on 1,000 permutations.

d $R^{2}$ is the proportion of phenotypic variance explained by the QTL.

e Additive effect for each QTL; a negative sign indicates a QTL effect from Kenya Kongoni.

f AUDPC for leaf rust at El Batán, 2011.

g AUDPC for leaf rust at Ciudad Obregón, 2011-12.

h LR12U leaf rust at La Estanzuela, 2011-12.

i AUDPC for leaf rust at Ciudad Obregón, 2012-13.

j AUDPC for yellow rust at Toluca, 2011.

k AUDPC for yellow rust at Toluca, 2012. 
A $1 B L$

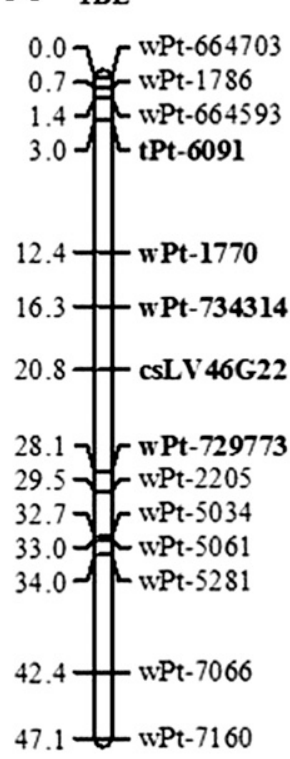

LOD

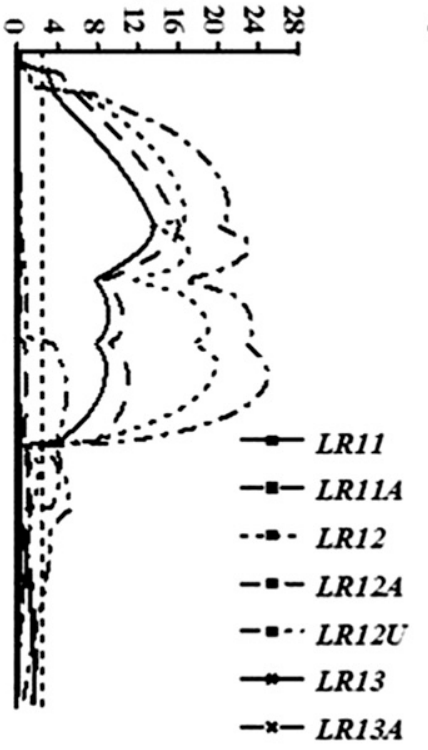

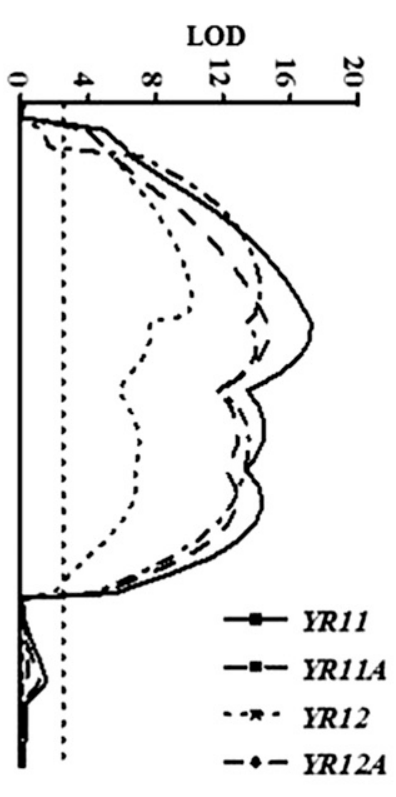

C
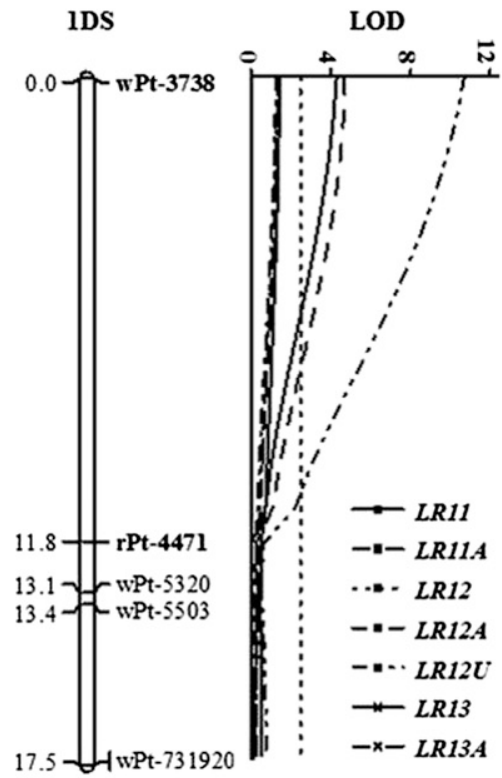

B

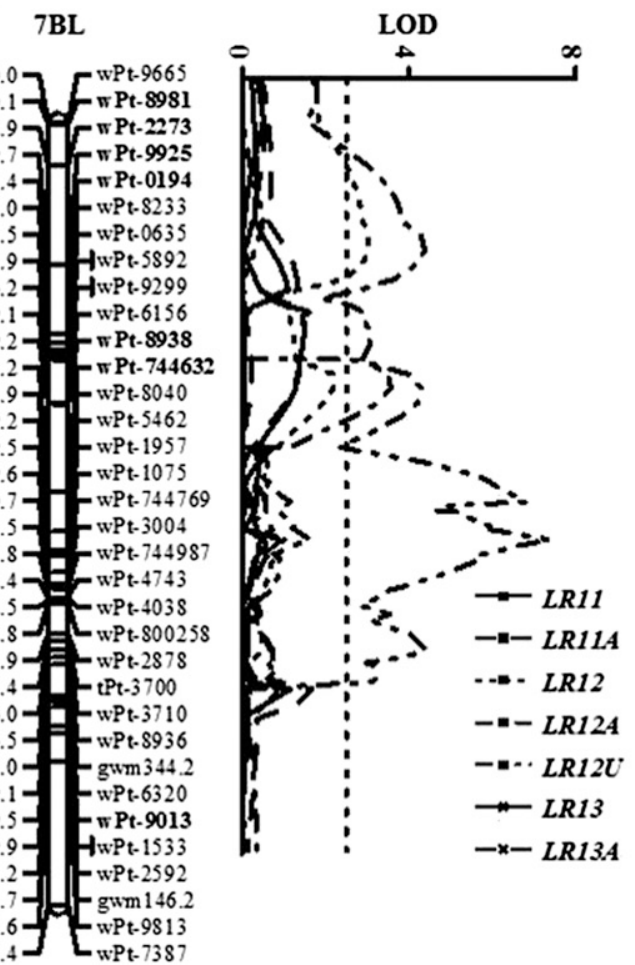

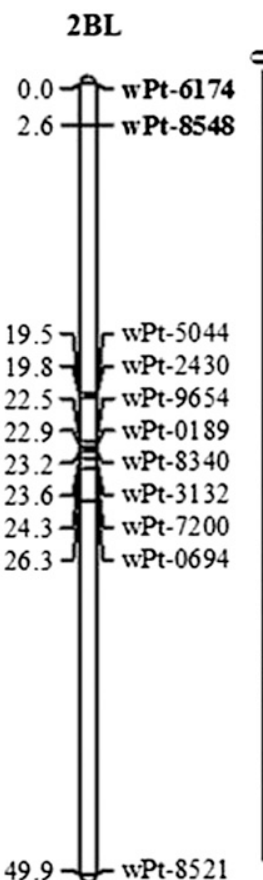

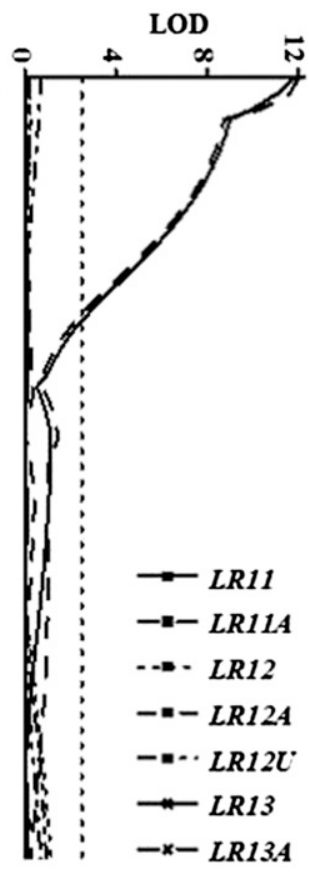

Continued on next page

Fig. 2. Likelihood plots of quantitative trait loci (QTL) for adult plant resistance to leaf rust (LR) on chromosomes A, 1BL; B, 7BL; C, 1DS; D, 2BL; and E, 3BS and to yellow rust (YR) on chromosomes A, 1BL; B, 7BL; F, 2AS; G, 2BS; and H, 5BL identified by QTL Cartographer in an Avocet-YrA/Kenya Kongoni recombinant inbred line population. The significant logarithm of odds (LOD) thresholds were detected based on 1,000 permutations. Positions (in centimorgans) of the molecular markers along each chromosome are shown on the vertical axes; cumulated genetic distances of linkage group are also given. LR11, LR12, and LR13 represent LR phenotypic data in Mexico (El Batán, 2011; Ciudad Obregón 2012-13) and Uruguay (La Estanzuela, 2012). YR11 and YR12 represent YR phenotypic data for Mexico (Toluca, 2011 and 2012). LR11A, LR12A, LR13A, LR13UA, YR11A, and YR12A represent area under the disease progress curve for LR in Mexico (El Batán, 2011 and Ciudad Obregón 2012-13) and Uruguay (La Estanzuela, 2012) and for YR in Mexico (Toluca, 2011 and 2012).

Another small effect locus conferring resistance to YR was found on chromosome 5BL (QYr.cim-5BL) between the markers Xbarc59 and $w P t-3922$. Chu et al. (6) identified an APR QTL, QLr.fcu$5 B L$, on the same chromosome between SSR markers Xgdm116Xbarc59 with a small effect on LR resistance and which explained about $7 \%$ of the phenotypic variation. In our study, the phenotypic variation for YR resistance ranged from 5 to $9 \%$. Interestingly, the same marker, Xbarc59, was linked to resistance loci in both studies.
Kenya Kongoni, released in Kenya in 1981, has remained resistant to LR and YR in Mexico, Uruguay, and Kenya. Our study shows that the resistance to LR and YR is controlled by four to five slow-rusting APR loci. Because Kenya Kongoni does not possess seedling resistance, it can be a valuable parent in breeding programs to achieve durable resistance to both rusts by crossing with other elite germplasm and pyramiding the resistance loci into a single cultivar using the closely linked molecular markers. 


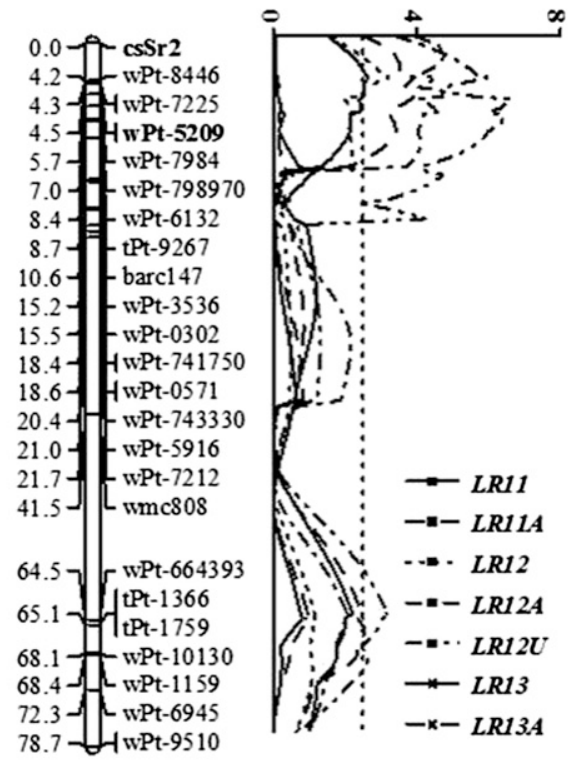

2AS

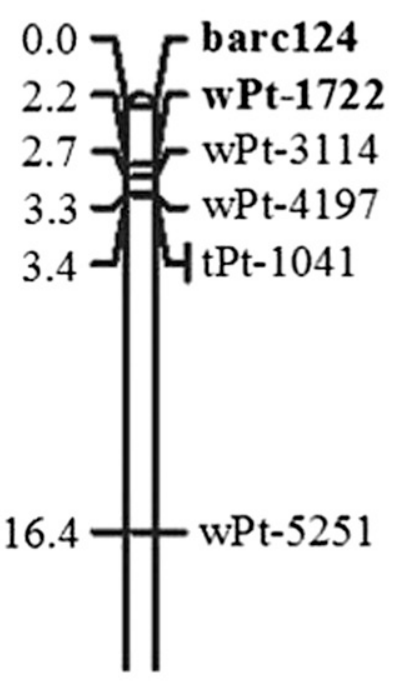

$\mathbf{F}$ LOD

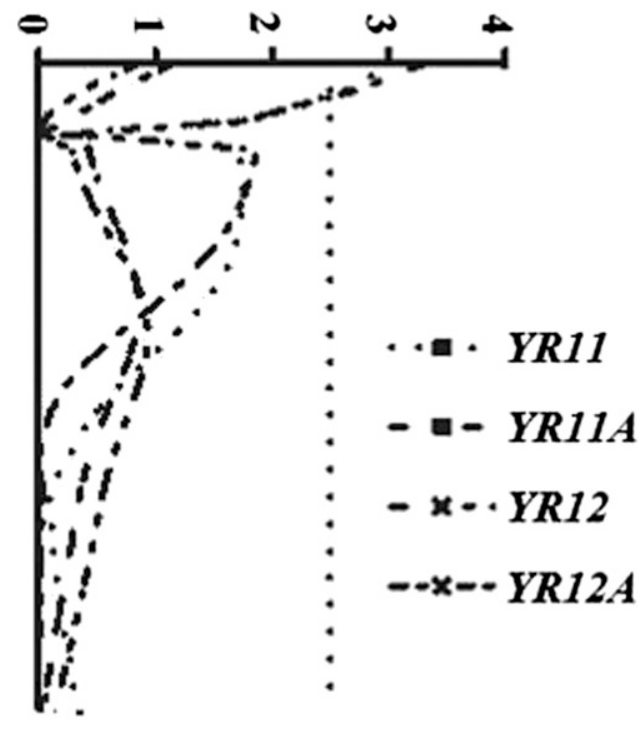

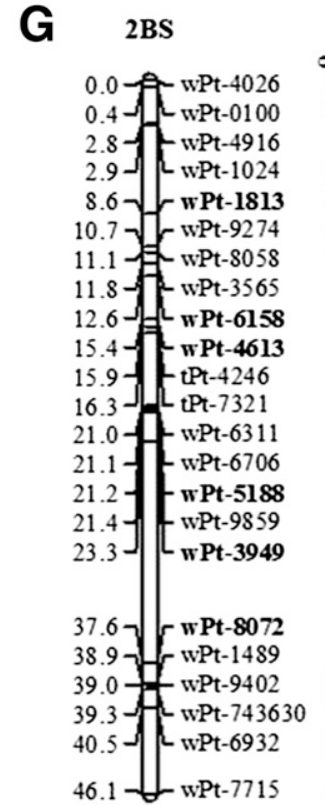

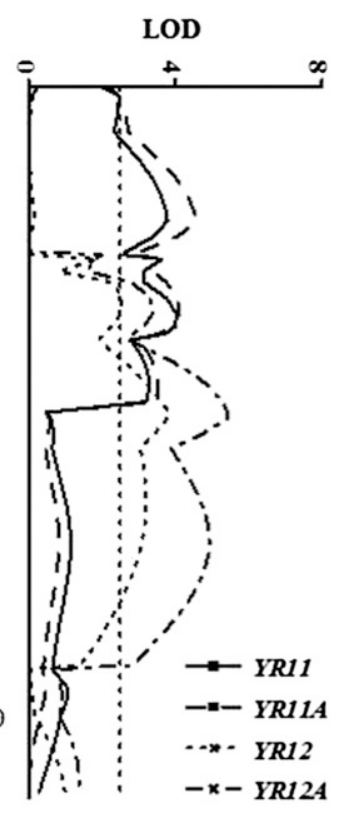

H

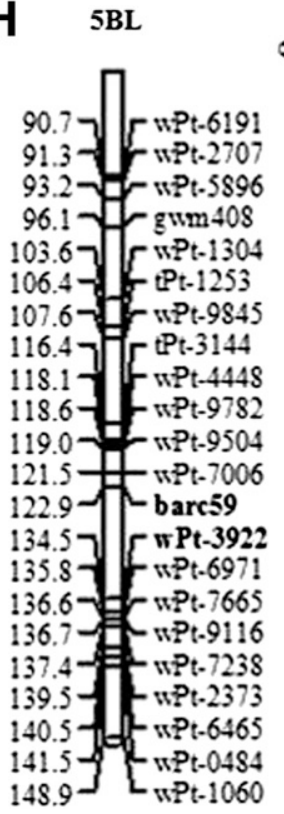

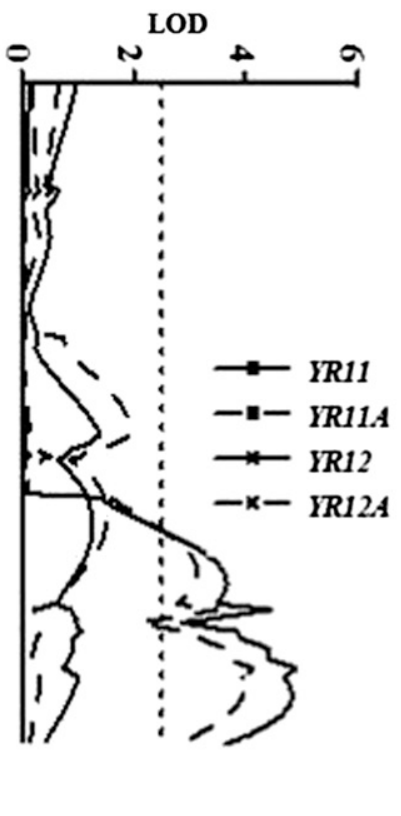

Fig. 2. Continued from preceding page

\section{Acknowledgments}

This work was supported by the Australian Grains Research and Development Corporation (GRDC) and Australian Cereal Rust Control Program. We thank M. Listman of CIMMYT for technical editing.

\section{Literature Cited}

1. Agenbag, G. M., Pretorius, Z. A., Boyd, L. A., Bender, C. M., and Prins, R. 2012. Identification of adult plant resistance to yellow rust in the cultivar Cappelle-Desprez. Theor. Appl. Genet. 125:109-120.

2. Bariana, H. S., Bansal, U. K., Schmidt, A., Lehmensiek, A., Kaur, J., Miah, H., Howes, N., and McIntyre, C. L. 2010. Molecular mapping of adult plant yellow rust resistance in wheat and identification of pyramided QTL genotypes. Euphytica 176:251-260.

3. Basnet, B. R., Singh, R. P., Ibrahim, A. M. H., Herrera-Foessel, S. A., HuertaEspino, J., Lan, C. X., and Rudd, J. C. 2014. Characterization of Yr54 and other genes associated with adult plant resistance to yellow rust and leaf rust in common wheat Quaiu 3. Mol. Breed. 33:385-399.

4. Bjarko, M. E., and Line, R. F. 1988. Quantitative determination of the gene action of leaf rust resistance in four cultivars of wheat Triticum aestivum. Phytopathology 78:451-456.
5. Chhuneja, P., Kaur, S., Garg, T., Ghai, M., Kaur, S., Prashar, M., Bains, N. S., Goel, R. K., Keller, B., Dhaliwal, H. S., and Singh, K. 2008. Mapping of adult plant yellow rust resistance genes in diploid A genome wheat species and their transfer to bread wheat. Theor. Appl. Genet. 116:313-324.

6. Chu, C. G., Friesen, T. L., Xu, S. S., Faris, J. D., and Kolmer, J. A. 2009. Identification of novel QTLs for seedling and adult plant leaf rust resistance in wheat doubled haploid population. Theor. Appl. Genet. 119:263-269.

7. Crossa, J., Burgueño, J., Dreisigacker, S., Vargas, M., Herrera-Foessel, S. A., Lillemo, M., Singh, R. P., Trethowan, R., Warburton, M., Franco, J., Reynolds, M., Crouch, J. H., and Ortiz, R. 2007. Association analysis of historical bread wheat germplasm using additive genetic covariance of relatives and population structure. Genetics 177:1889-1913.

8. Dedryver, F., Paillard, S., Mallard, S., Robert, O., Trottet, M., Nègre, S., Verplancke, G., and Jahier, J. 2009. Characterization of genetic components involved in durable resistance to yellow rust in the bread wheat "Renan". Phytopathology 99:968-973.

9. Dixon, J., Braun, H. J., Kosina, P., and Crouch, J. 2009. Wheat Facts and Futures 2009. CIMMYT, D.F., Mexico.

10. Francki, M. G., Walker, E., Crawford, A. C., Broughton, S., Ohm, H. W., Barclay, I., Wilson, R. E., and McLean, R. 2009. Comparison of genetic 
and cytogenetic maps of hexaploid wheat (Triticum aestivum L.) using SSR and DArT markers. Mol. Genet. Genomics 281:181-191.

11. German, S., Barcellos, A., Chaves, M., Kohli, M., Campos, P., and de Viedma, L. 2007. The situation of common wheat rusts in the Southern Cone of America and perspectives for control. Aust. J. Agric. Res. 58:620-630.

12. German, S., Chaves, M., Campos, P., de Viedma, L., and Madariaga, R. 2009. Are rust pathogens under control in the Southern cone of South America. Page 65 in: Proc. Borlaug Global Rust Initiative, 2009 Tech. Workshop, Cd. Obregón, Sonora, Mexico.

13. Hao, Y. F., Chan, Z. B., Wang, Y. Y., Bland, D., Buck, J., Brown-Guedira, G., and Johnson, J. 2011. Characterization of a major QTL for adult plant resistance to yellow rust in US soft red winter wheat. Theor. Appl. Genet. 123:1401-1411

14. Herrera-Foessel, S. A., Singh, R. P., Huerta-Espino, J., Rosewarne, G. M., Periyannan, S. K., Viccars, L., Calvo-Salazar, V., Lan, C. X., and Lagudah, E. S. 2012. Lr68: A new gene conferring slow rusting resistance to leaf rust in wheat. Theor. Appl. Genet. 124:1475-1486.

15. Herrera Foessel, S. A., Singh, R. P., Lillemo, M., Huerta-Espino, J., Bhavani, S., Sukhwinder, S., Lan, C. X., Calvo-Salazar, V., and Lagudah, E. S. 2014. Lr67/Yr46 confers adult plant resistance to stem rust and powdery mildew in wheat. Theor. Appl. Genet. 127:781-789.

16. Huang, B. E., George, A. W., Forrest, K. L., Kilian, A., Hayden, M. J., Morell, M. K., and Cavanagh, C. R. 2012. A multiparent advanced generation intercross population for genetic analysis in wheat. Plant Biotechnol. J 10:826-839.

17. Huerta-Espino, J., and Singh, R. P. 2000. Wheat rust. Pages 231-249 in: The Temporal Wheat in Mexico. M. H. E. Villaseñor and R. E. Espitia, eds. Secretaría de Agricultura, Ganadería y Desarrollo Rural (SAGAR), Instituto Nacional de Investigaciones Forestales, Agrícolas y Pecuarias (INIFAP), Centro de Investigación Regional-Centro (CIR-CENTRO), Campo Experimental Valle de Mexico, CEVAMEX, Mexico.

18. Johnson, R. 1981. Durable resistance: Definition, genetic control, and attainment in plant breeding. Phytopathology 71:567-568.

19. Khlestkina, E. K., Roder, M. S., Unger, O., Meinel, A., and Borner, A. 2007. More precise map position and origin of a durable non-specific adult plant disease resistance against yellow rust (Puccinia striiformis) in wheat. Euphytica 153:1-10.

20. Knott, D. R., and Padidam, M. 1988. Inheritance of resistance to stem rust in six wheat lines having adult plant resistance. Genome 30:283-288.

21. Kolmer, J. A., Singh, R. P., Garvin, D. F., Viccars, L., William, H. M., HuertaEspino, J., Obonnaya, F. C., Raman, H., Orford, S., Bariana, H. S., and Lagudah, E. S. 2008. Analysis of the Lr34/Yr18 rust resistance region in wheat germplasm. Crop Sci. 48:1841-1852.

22. Krattinger, S. G., Lagudah, E. S., Spielmeyer, W., Singh, R. P., HuertaEspino, J., McFadden, H., Bossolini, E., Selter, L. L., and Keller, B. 2009. A putative $\mathrm{ABC}$ transporter confers durable resistance to multiple fungal pathogens in wheat. Science 323:1360-1363.

23. Lagudah, E. S. 2011. Molecular genetics of race non-specific rust resistance in wheat. Euphytica 179:81-91.

24. Lagudah, E. S., Krattinger, S. G., Herrera-Foessel, S., Singh, R. P., HuertaEspino, J., Spielmeyer, W., Brown-Guedira, G., Selter, L. L., and Keller, B. 2009. Gene-specific markers for the wheat gene. Theor. Appl. Genet. 119: 889-898.

25. Lan, C. X., Singh, R. P., Huerta-Espino, J., Calvo-Salazar, V., and HerreraFoessel, S. A. 2014. Genetic analysis of resistance to leaf rust and yellow rust in wheat cultivar Francolin\#1. Plant Dis. 98:1227-1234.

26. Li, Z. F., Lan, C. X., He, Z. H., Singh, R. P., Rosewarne, G. M., Chen, X. M., and Xia, X. C. 2014. Overview and application of QTL for adult plant resistance to leaf rust and powdery mildew in wheat. Crop Sci. 54:1907-1925.

27. Lin, F., and Chen, X. M. 2007. Genetics and molecular mapping of genes for race-specific all-stage resistance and non-race specific high-temperature adultplant resistance to yellow rust in spring wheat cultivar Alpowa. Theor. Appl. Genet. 114:1277-1287.

28. Mago, R., Brown-Guedira, G., Dreisigacker, S., Breen, J., Jin, Y., Singh, R., Appels, R., Lagudah, E. S., Ellis, J., and Spielmeyer, W. 2011. An accurate DNA marker assay for stem rust resistance gene $\mathrm{Sr} 2$ in wheat. Theor. Appl. Genet. 122:735-744.

29. Mallard, S., Gaudet, D., Aldeia, A., Abelard, C., Besnard, A. L., Sourdille, P., and Dedryver, F. 2005. Genetic analysis of durable resistance to yellow rust in bread wheat. Theor. Appl. Genet. 110:1401-1409.

30. McIntosh, R. A., Dubcovsky, J., Rogers, W. J., Morris, C., Appels, R., and Xia, X. C. 2014. Catalogue of gene symbols for wheat: 2013-2014 (supplement). Online publication. http://www.shigen.nig.ac.jp/wheat/komugi/genes/macgene/ supplement2013.pdf

31. Mulitze, D. K., and Baker, R. J. 1985. Genotype assay and method of moments analysis of five quantitative traits in a spring wheat cross. Crop Sci. 25:162-167.

32. Peterson, R. F., Campbell, A. B., and Hannah, A. E. 1948. A diagrammatic scale for estimating rust intensity on leaves and stems of cereals. Can. J. Res. 26c:496-500.

33. Rodríguez-García, M. F., Huerta-Espino, J., Villaseñor-Mir, H. E., and SolísMoya, E. 2009. Virulence of the wheat yellow rust in the main irrigated production areas of Mexico. Agric. Tcc. Mex. 35:179-187.

34. Roelfs, A. P., Singh, R. P., and Saari, E. E. 1992. Rust Diseases of Wheat: Concepts and Methods of Disease Management. CIMMYT, D.F., Mexico.
35. Rosewarne, G. M., Herrera-Foessel, S. A., Singh, R. P., Huerta-Espino, J. Lan, C. X., and He, Z. H. 2013. Quantitative trait loci of yellow rust resistance in wheat. Theor. Appl. Genet. 126:2427-2449.

36. Rosewarne, G. M., Singh, R. P., Huerta-Espino, J., Herrera-Foessel, S. A., Forrest, K. L., Hayden, M. J., and Rebetzke, G. J. 2012. Analysis of leaf and yellow rust severities reveals pathotype changes and multiple minor QTLs associated with resistance in Avocet $\times$ Pastor wheat population. Theor. Appl. Genet. 124:1283-1294

37. Rosewarne, G. M., Singh, R. P., Huerta-Espino, J., and Rebetzke, G. J. 2008 Quantitative trait loci for slow rusting resistance in wheat lo leaf rust and yellow rust identified with multi-environment analysis. Theor. Appl. Genet. 116:1027-1034.

38. Sayre, K. D., Singh, R. P., Huerta-Espino, J., and Rajaram, S. 1998. Genetic progress in reducing losses to leaf rust in CIMMYT-derived Mexican Spring wheat cultivars. Crop Sci. 38:654-659.

39. Singh, R. P., Herrera-Foessel, S. A., Huerta-Espino, J., Bariana, H. S., Bansal, U., McCallum, B. D., Hiebert, C. W., Bhavani, S., Singh, S., Lan, C. X., and Lagudah, E. S. 2012. Lr34/Yr18/Sr57/Pm38/Bdv1/Ltn1 confers slow rusting, adult plant resistance to Puccinia graminis tritici. Page 173 in: 13th Cereal Rust Powdery Mildew Conf. Friendship Hotel, Beijing.

40. Singh, R. P., Herrera-Foessel, S. A., Huerta-Espino, J., Lan, C. X., Basnet, B. R., Bhavani, S., and Lagudah, E. S. 2013. Pleiotropic gene Lr46/Yr29/Pm39/ Ltn2 confers slow rusting, adult plant resistance to wheat stem rust fungus. Page 17.1 in: Proc. Borlaug Global Rust Initiative, 2013 Tech. Workshop, New Delhi, India.

41. Singh, R. P., Huerta-Espino, J., Bhavani, S., Herrera-Foessel, S. A., Singh, D. Singh, P. K., Velu, G., Mason, R. E., Jin, Y., Njau, P., and Crossa, J. 2011 Race-nonspecific resistance to rust diseases in CIMMYT spring wheats. Euphytica 179:175-186.

42. Singh, R. P., Mujeeb-Kazi, A., and Huerta-Espino, J. 1998. Lr46: A gene conferring slow-rusting to leaf rust in wheat. Phytopathology 88:890-894.

43. Singh, R. P., Nelson, J. C., and Sorrel, M. E. 2000. Mapping Yr28 and other genes for resistance to yellow rust in wheat. Crop Sci. 40:1148-1155.

44. Singh, R. P., and Rajaram, S. 1992. Genetics of adult-plant resistance to leaf rust in "Frontana" and three CIMMYT wheats. Genome 35:24-31.

45. Singh, R. P., William, H. M., Huerta-Espino, J., and Crosby, M. 2003 Identification and mapping of gene $\operatorname{Yr} 31$ for resistance to stripe rust in Triticum aestivum cultivar Pastor vol 1. Pages 411-413 in: Proc. 10th Int. Wheat Genet. Symp. N. E. Pogna, M. Romana, E. A. Pogna, and G Galterio, eds. Instituto Sperimentale per la Cerealicoltura, Rome.

46. Somers, D. J., Isaac, P., and Edwards, K. 2004. A high density microsatellite consensus map for bread wheat (Triticum aestivum L.). Theor. Appl. Genet 109:1105-1114.

47. Spielmeyer, W., Sharp, P. J., and Lagudah, E. S. 2003. Identification and validation of markers linked to broad-spectrum stem rust resistance gene Sr2 in wheat (Triticum aestivum L.). Crop Sci. 43:333-336.

48. Suenaga, K., Singh, R. P., Huerta-Espino, J., and William, H. M. 2003 Microsatellite markers and genes $\mathrm{Lr} 34 / \mathrm{Yr} 18$ and other quantitative trait loci for leaf rust and stripe rust resistance in bread wheat. Phytopathology 93 : 881-889.

49. Sun, X., Bowden, R., Caver, B. F., and Bai, G. 2010. Molecular mapping of wheat leaf rust resistance gene $L r 42$. Crop Sci. 50:59-66.

50. Uauy, C., Brevis, J. C., Chen, X., Khan, I., Jackson, L., Chicaiza, O., Distelfeld, A., Fahima, T., and Dubcovsky, J. 2005. High-temperature adultplant (HTAP) yellow rust resistance gene Yr36 from Triticum turgidum ssp. dicoccoides is closely linked to the grain protein content locus Gpc-B1. Theor. Appl. Genet. 112:97-105.

51. Van Ooijen, J. W. 2006. JoinMap 4, Software for the Calculation of Genetic Linkage Maps in Experimental Populations. Plant Research International and Kyazma B.V., Wageningen, The Netherlands.

52. Vazquez, M. D., Peterson, C. J., Riera-Lizarazu, O., Chen, X., Heesacker, A., Ammar, K., Crossa, J., and Mundt, C. C. 2012. Genetic analysis of adult plant, quantitative resistance to stripe rust in wheat cultivar 'Stephens' in multienvironment trials. Theor. Appl. Genet. 124:1-11.

53. Voorrips, R. E. 2002. MapChart: Software for the graphical presentation of linkage maps and QTLs. Heredity 93:77-78.

54. Wang, S., Basten, C. J., and Zeng, Z. B. 2012. Windows QTL Cartographer 2.5. Department of Statistics, North Carolina State University, Raleigh Online publication. http://statgen.ncsu.edu/qtlcart/WQTLCart.htm

55. Wellings, C. R. 1992. Resistance to stripe (yellow) rust in selected spring wheats. Vor. Pflan. 24:273-275.

56. Wilkinson, A. P., Winfield, O. M., Baker, L. A., Allen, M. A., Burridge, A., Coghill, A. J., and Edward, J. K. 2012. CerealsDB 2.0: An integrated resource for plant breeders and scientists. BMC Bioinf. 13:219.

57. William, H. M., Singh, R. P., Huerta-Espino, J., Palacios, G., and Suenaga, K 2006. Characterization of genetic loci conferring adult plant resistance to leaf rust and stripe rust in spring wheat. Genome 49:977-990.

58. William, M., Singh, R. P., Huerta-Espino, J., Islas, S. O., and Hoisington, D. 2003. Molecular marker mapping of leaf rust resistance gene Lr46 and its association with stripe rust resistance gene $Y r 29$ in wheat. Phytopathology 93:153-159

59. Wright, S. 1968. Evolution and the genetics of populations. In: Genetic and Biometric Foundations, Vol. 1. The University of Chicago Press, Chicago. 